\title{
High Performance Surface Plasmon Resonance Based Photonic Crystal Fiber Sensor with Four Open Surface Rings
}

neda Ali porghoveh ( $\nabla$ nedaporghoveh@yahoo.com )

University of Tabriz https://orcid.org/0000-0002-6376-840X

Jamal Barvestani

University of Tabriz

Bahar Meshginqalam

University of Tabriz

\section{Research Article}

Keywords: Photonic crystal fiber sensor, Surface plasmon resonance, Confinement Loss, Amplitude sensitivity, Fundamental mode, Refractive index.

Posted Date: July 30th, 2021

DOI: https://doi.org/10.21203/rs.3.rs-679061/v1

License: (9) This work is licensed under a Creative Commons Attribution 4.0 International License. Read Full License 


\title{
High performance surface plasmon resonance based photonic crystal fiber sensor with four open surface rings
}

\author{
Ali-Porghoveh Neda*, Barvestani Jamal, Meshginqalam Bahar \\ Faculty of Physics, University of Tabriz, Tabriz, Iran \\ *nedaporghoveh@yahoo.com
}

\begin{abstract}
In this paper, a photonic crystal fiber sensor is proposed which have four-opening channels that support surface plasmon resonances. By detecting the optical spectrum of leakage from the fiber core to the four regions created on its periphery, the sensitivity of the sensor can be assessed. Gold is used as the plasmonic material in the infrared wavelength range on the Titanium oxide film which is used as a substrate layer to increase the coupling and adhesion. Influences of structural parameters on the sensor performance are investigated by the Finite Difference Element method. Sensitivities for refractive index of 1.34-1.44 for wavelength ranges (690-1500 nm) with maximum wavelength sensitivity of 25,600 nm / RIU and maximum amplitude sensitivity and $7367\left(\mathrm{RIU}^{-1}\right)$ and figure of merit (FOM) of 547 ( $\mathrm{RIU}^{-1}$ ) have been calculated. In addition to high sensitivity, great FOM value of this sensor is an important feature. This sensor can be used to identify chemicals and biomolecules.
\end{abstract}

Keywords: Photonic crystal fiber sensor; Surface plasmon resonance; Confinement Loss; Amplitude sensitivity; Fundamental mode; Refractive index.

\section{Introduction}

Due to the demand and need for optical sensors, especially for medical applications as well as the implementation of sensors within the integrated equations, downsizing and increasing the sensitivity of sensors in small dimensions has become very important [1-2]. One of the sensing mechanisms in the identification of chemicals and biomolecules as well as liquids is refractive index based sensor, wavelength based sensor or wavelength based sensor or amplitude based sensor [2]. In this regard, such optical fiber sensors will be designed and manufactured by methods such as Bragg interferometry and grating to monitor water pollution, detect chemicals, and so on [3-4].

In last two decades, surface plasmon resonance (SPR) has been used in the development of unlabeled optical sensors with high sensitivity [5]. Researches have been done on the application of SPR-based optical sensors using different structures and methods. Surface plasmon amplification is an electromagnetic mode in which an optical electric field with TM polarization is coupled at the boundary between a dielectric and a metal surface by electron oscillation, and two waves intensify, a type of two-wave interference that occurs constructively. Increases the intensity of the electric field and can improve the sensor performance of an optical sensor. During Optical waves, metals such as gold, silver, aluminum are used to intensify the surface plasmon, but the reason for this choice is the lower losses in this metal than other metals. Many metals at optical frequencies have a negative dielectric constant that causes high surface reflectance [5-6]. That, surface plasmon resonance has been used in the development of high-sensitivity optical sensing. For this purpose, a part of the fiber sheath is cut in the form of a fading field to the surrounding environment on the surface of the sheath, coupled and leaked out. Using this fading wave, a change is made in the refractive index of the external fluid that penetrates into the grooves created. The dielectric boundary and the coated metal are provided. If there is a change in the refractive index of the environment around the metal layer, it causes a change in the fuzzy coupling condition. Fraction coefficient changes can be detected by changing the coupling conditions that cause the frequency spectrum to shift.

till now, different optical fiber sensors based on SPR have been reported such as conventional fiber [6], fiber Bragg grating [7], photonic quasi-crystal fiber (PQF) [8], and photonic crystal fiber (PCF) [9]. PQF because of low loss [10], numerous birefringence and low nonlinearity [11], rich double-cladding features [12] is not important. Therefore, much endeavor has been centralized on SPR based PCF sensors (PCF-SPR) because realize miniaturization and system integration. PCF is a kind of micro-structured optical fiber with distinct advantages including the tunable effective refractive index in the fiber core, birefringence, large mode area, and flat dispersion. [13]. Compared with SPR based conventional optical fiber sensors [14], the sensitivity of PCF-SPR sensors is two orders of magnitude higher [15]. The various PCF-SPR sensors have been designed. For instance, Paul et al. 
described a dual-core PCF-SPR sensor [16]. Tong et al. and Dash and et al. designed a D-shaped PCF-SPR sensor with the sensitivity of $4850 \mathrm{~nm} / \mathrm{RIU}$ and $5200 \mathrm{~nm} / \mathrm{RIU}$, respectively [17-18]. M. Hautakorpi described a three-hole micro-structured sensor [19].

To achieve high performance of PCF-SPR sensors, surface engineering of the structure has a vital role. In this work, a novel PCF-SPR sensor with four opening surface channels is proposed. $\mathrm{TiO}_{2}$ and gold are chosen as the plasmonic materials, where the gold layer deposited on the $\mathrm{TiO}_{2}$ layer to improve the sensitivity. By optimizing the parameters of structure such as position and radius of surface rings, first air hole radius and thicknesses of gold and $\mathrm{TiO}_{2}$ films, a maximum wavelength sensitivity of $25,600 \mathrm{~nm} / \mathrm{RIU}$ is attained for analyte RIs between 1.34 and 1.44. Our designed sensor can better meet the request for a wide detection of various analyte and may have mighty potential in optical sensing applications.

\section{Design and method}

The schematic diagram of the PCF-SPR sensor that is created of four-opening channels coated with $\mathrm{TiO}_{2}$ and gold films as plasmonic materials is presented in figure 1. Three hexagonal rows of air holes stacked in which some rotations have done and four holes of the second row are removed to obtain good coupling between fundamental core mode and those appeared at the surface of the four opening rings. Radii of holes of second and third rows are assumed to be, $r_{2}=r_{3}=0.65 \mu \mathrm{m}$. The distance from the center of the first ring of air holes to the core was at $2 \mu \mathrm{m}$, which is defined as pitch $(\Lambda)$. The another two pitches were fixed as $1.4 \Lambda$ and $1.8 \Lambda$ for the distance from the second- and third-ring air holes to the core, respectively. Radii of the cladding air holes of the first ring was $\mathrm{R}_{1}=1.34 \mu \mathrm{m}$ and Radii of the other air holes was selected to be, $\mathrm{R}_{2}=\mathrm{R}_{3}=1.3 \mu \mathrm{m}$. These big four-opening channels are more conducive to the deposition of metal thin films which support SPR modes. The radii of the open ring channels are $1 \mu \mathrm{m}$ and the distance of the center of these rings to the center of the fiber is $R_{H}=4 \mu \mathrm{m}$. Gold and $\mathrm{TiO}_{2}$ layers with the thicknesses $\mathrm{t}_{\mathrm{Au}}$ and $\mathrm{t}_{\mathrm{TiO}_{2}}$ are deposited at the inner surface of the open rings.

\section{Fig. 1.}

The background material of the sensor is silica glass, in which the dispersion relation is followed by Sellmeier equation [20]:

$$
\mathrm{n}^{2}(\lambda)=1+\frac{B_{1} \lambda^{2}}{\lambda^{2}-C_{1}}+\frac{B_{2} \lambda^{2}}{\lambda^{2}-C_{2}}+\frac{B_{3} \lambda^{2}}{\lambda^{2}-C_{3}}
$$

where $\mathrm{n}$ is the RI of the fused silica, which is dependent on wavelength $\lambda . \mathrm{B}_{1}, \mathrm{~B}_{2}, \mathrm{~B}_{3}, \mathrm{C}_{1}, \mathrm{C}_{2}$, and $\mathrm{C}_{3}$ are known as the Sellmeier constants. The values of these constants for the fused silica are: $0.69616300,0.407942600$, $0.897479400,0.00467914826,0.0135120631$ and 97.9340025 , respectively.

The permittivity of gold is given by Drude-Lorenz model [20].A thin layer of $\mathrm{TiO}_{2}$ is also used between gold and silica, which assists in reducing the adhesion problem of $\mathrm{Au}$ and improves the sensitivity. This layer is also helpful for exciting the SPR with efficiently contacting the core-guided mode to the SPR mode [21]. The dielectric constant of titanium oxide is calculated by the following equation [22]:

$\mathrm{n}^{2} \mathrm{TiO}_{2}(\lambda)=5.913+\frac{2.441 \times 10^{7}}{\lambda^{2}-0.803 \times 10^{7}}$

where $\lambda$ is the wavelength measured in $\mu \mathrm{m}$ and $\mathrm{n}_{\mathrm{TiO}_{2}}$ is the wavelength-dependent RI of titanium oxide.

The confinement loss is a crucially important performance parameter for the PCF-SPR sensor, which can be determined by equation [23]:

$\alpha(\lambda)=8.686 \times \mathrm{k}_{0} \times \operatorname{Im}\left(\mathrm{n}_{\mathrm{eff}}\right) \times 10^{4}\left(\frac{d B}{c m}\right)$

where the imaginary effective mode index is denoted as $\operatorname{Im}\left(\mathrm{n}_{\mathrm{eff}}\right)$, the wave number is $K_{0}=\frac{2 \pi}{\lambda}$, and $\lambda$ is the operating wavelength. Analyte sensing happen with variations of the wavelength in the surrounding environment for the biotargets.

The wavelength sensitivity is another the important performance parameter of the PCF sensor, which can be calculated by equation [24]: 
$\mathrm{S}_{\mathrm{w}}=\frac{\Delta \lambda_{\text {peak }}}{\Delta \mathrm{n}_{\mathrm{a}}}(\mathrm{nm} / \mathrm{RIU})$

In addition, how to calculate the sensitivity of the amplitude is through the following formula [24].

$\mathrm{S}_{\mathrm{A}}=-\frac{1}{\alpha\left(\lambda, \mathrm{n}_{\mathrm{a}}\right)} \frac{\partial \alpha\left(\lambda, \mathrm{n}_{\mathrm{a}}\right)}{\partial \mathrm{n}_{\mathrm{a}}}\left(\mathrm{RIU}^{-1}\right)$

Also, the figure of merit (FOM), which provides sensitivity and Full width at half maximum (FWHM), is obtained by equation [25]:

$\mathrm{FOM}=\frac{\mathrm{S}_{\mathrm{W}}}{\mathrm{FWHM}}\left(\mathrm{RIU}^{-1}\right)$

\section{Results and discussion}

This section presents numerical result of proposed SPR based photonic crystal fiber for different parameters of the structure. Figure 2, shows the dispersion of fundamental core and SPR modes. The dotted (solid) line shows the dispersion of real (imaginary) part of the effective refractive index of fundamental mode and dashed line displays the dispersion of SPR mode. The SPR and core-guided modes intersected at wavelength of $1.16 \mu \mathrm{m}$, and a peak was observed at the point of intersection, where the phase matching condition is satisfied for the analyte RI of 1.43 . At such a point, the core mode and the plasmonic mode are coupled. At the resonance wavelength, a sharp loss peak was observed, and unknown samples could be effectively determined by shifting this peak to a longer or shorter wavelength for different analyte refractive indices.

Fig. 2.

The loss peaks for two analytes with refractive indices of 1.41 and 1.42 are shown in figure 3 . For the case of 1.41 , the loss peak occurred at the wavelength of $0.966 \mu \mathrm{m}$ and correspondingly for the case of 1.42 , it takes wavelength of $1.085 \mu \mathrm{m}$. Thus, according to equation (4), the wavelength shift between two analytes shows the wavelength sensitivity of $11,900 \mathrm{~nm} / \mathrm{RIU}$.

Fig. 3.

In order to investigate the effect of numerous structural parameters on sensor performance and select the optimized values, all of the parameters are classified and explained in the following figures and tables. These calculations are done considering analyte RI variations of 1.43-1.44.

To investigate the effects of $\mathrm{TiO}_{2}$ thicknes on the performance of the proposed sensor, we have showed the sensitivities and FOM values versus the thickness of $\mathrm{TiO}_{2}$ film in figure 4, in which the gold thickness is $30 \mathrm{~nm}$ and the radius of air cavities are supposed to be $0.6 \mathrm{~nm}$. These results show that the highest wavelength sensitivity and FOM as $23,000 \mathrm{~nm} / \mathrm{R} 1 \mathrm{U}$ and $466.66\left(\mathrm{RIU}^{-1}\right)$, that take place at $\mathrm{t}_{\mathrm{TiO}_{2}}=11 \mathrm{~nm}$. Also this figure indicates the positive effect of the $\mathrm{TiO}_{2}$ layer presence on both sensitivity and FOM values. Thus, we assumed, $t_{\mathrm{TiO}_{2}}=11 \mathrm{~nm}$ as an optimum value in the rest of the paper.

Fig. 4.

Similarly, in figure 5, the sensor performance in terms of sensitivity and FOM is plotted for different Au thicknesses.

Fig. 5. 
As it is clear from figure 5, the maximum values of wavelength sensitivity and FOM relate to the $t_{A u}=40 \mathrm{~nm}$. It should be mentioned that the corresponding values are $21,700 \mathrm{~nm} / \mathrm{R} 1 \mathrm{U}$ and $391.66\left(\mathrm{RIU}^{-1}\right)$, respectively.

Finally, in figure 6, the sensor performance using of previous optimum values are examined for different values of the radius of the first row of air holes. It is seen from this figure and respect to the maximum values of the sensitivity and FOM, $r_{1}=0.67 \mu \mathrm{m}$ is the optimum value.

Fig.6. (a).

Now we are in a position to plot the loss spectra of the proposed PCF-SPR sensor for various analyte RI by using of optimized values. Loss spectra are presented in figure 7 for the wide range of analyte RI.

Fig. 7.

Loss peaks have red shifts behavior and we can conclude that the proposed sensor depicts good sensitivity at higher refractive indices. To quantitatively show these behaviors, we have summarized the corresponding sensing parameters in table 1 .

Also, the amplitude sensitivities of the sensor are added in this table. To get better insight, we have shown corresponding amplitude sensitivities in figure 8 . We see that, the proposed sensor shows the amplitude sensitivities for the wide range of RI of analytes.

Table 1. Performance of proposed sensor for the analyte RI detection range from 1.34 to 1.44 .

\begin{tabular}{lccc}
\hline $\begin{array}{c}\Delta \boldsymbol{n}_{\boldsymbol{a}} \\
(\mathbf{R I U})\end{array}$ & $\begin{array}{c}\mathbf{S}_{\mathbf{W}} \\
(\mathbf{n m} / \mathbf{R I U})\end{array}$ & $\begin{array}{c}\mathbf{S}_{\mathbf{A}} \\
\left(\mathbf{R I U}^{\mathbf{1}}\right)\end{array}$ & $\begin{array}{c}\mathbf{F O M} \\
\left(\mathbf{R I U}^{-\mathbf{1}}\right)\end{array}$ \\
\hline $1.34-1.35$ & & & \\
$1.35-1.36$ & 1000 & 68.74 & 20.70 \\
$1.36-1.37$ & 1000 & 100.2 & 21.39 \\
$1.37-1.38$ & 2000 & 131.6 & 41.24 \\
$1.38-1.39$ & 2000 & 181 & 39.34 \\
$1.39-1.40$ & 3000 & 254 & 59.38 \\
$1.40-1.41$ & 4000 & 393.1 & 73.07 \\
$1.41-1.42$ & 7000 & 636.2 & 106.83 \\
$1.42-1.43$ & 11000 & 1001 & 152.77 \\
$1.43-1.44$ & 18000 & 2636 & 230.32 \\
& 25600 & 7367 & 547
\end{tabular}

Fig. 8.

It can be evident from the figure that the amplitude sensitivity increases gradually with an increase of analyte RI and reaches the maximum value for analyte RI of 1.44 which are $7367\left(\mathrm{RIU}^{-1}\right)$.

At the end, to show the performance of the proposed sensor, FOM is plotted versus the analyte RI variations in figure 9 .

\section{Fig. 9.}

As the RI of the analyte increases, the loss curve becomes sharper. This type of change indicates the decreasing of FWHM which results to the FOM increment. We obtain the maximum FOM as 547 (RIU ${ }^{-1}$ ) for the case of 1.431.44 range. This FOM is higher than the similar work reported in recent articles [26].

\section{Conclusions}


In this paper, we investigate a novel design of PCF-SPR sensors with four-opening channels for an analyte sensing range between 1.34 and 1.44. The sensing performance of the proposed sensor is analyzed numerically by Finite Difference Element method (FDE). The simulation results show a maximum wavelength sensitivity of 25,600 $\mathrm{nm} / \mathrm{RIU}$, and a low propagation loss. The maximum figure of merit (FOM) of 547 ( $\mathrm{RIU}^{-1}$ ), is achieved within the analyte RI range of 1.34 to 1.44 for this design which is better than the reported existing works. In addition, the maximum amplitude sensitivity of $7367\left(\mathrm{RIU}^{-1}\right)$ is calculated. Because of the ultra-low loss and high wavelength sensitivity and amplitude sensitivity, the proposed sensor can be a promising candidate for application in medical, detecting various bio-samples in the lab-on-a-fiber platform and environmental monitoring.

Funding: The research has been supported by the university of Tabriz

Conflicts of interest/Competing interests: Not applicable

Availability of data and material: Data and content were simulated with Lumerical software.

Code availability: This is simulated with Lumerical software.

Authors' contributions: The authors are written in order of work.

Ethics approval: Yes, I am satisfied.

Consent to participate: Yes, I am satisfied.

Consent for publication: Yes, I am satisfied.

\section{References}

[1] A. K. Sharma, A. K. Pandey, B. A Kaur, Review of advancements (2007-2017) in plasmonics- based optical fiber sensors. Opt. Fiber Technol. 43 (2018), 20-34.

[2] M. F. Azman, G. A. Mahdirazi, W. R. Wong, R. A. Aoni, F. M. R. Adikan, Design and fabrication of copperfilled photonic crystal fiber based polarization filters. Appl. Opt. 58 (2019), 2068-2075.

[3] L. Zao, H. Han, Y. Lian, N. Luan, J. Liu, Theoretical analysis of all-solid D-type photonic crystal fiber based plasmonic sensore for refractive index and temperature sensing. Opt. Fiber Technol. 50 (2019), 165-171.

[4] M. R. Momota, M. R. Hasan, Hollow-core silver coated photonic crystal fiber plasmonic sensor. Opt. Mater. 76 (2018) 287-294.

[5] B. Meshginqalam, J. Barvestani, Performance Enhancement of SPR Biosensor Based on Phosphorene and Transition Metal Dichalcogenides for Sensing DNA Hybridization. IEEE SENSORS JOURNAL 18 (18) (2018), 7537.

[6] T. Khanikar, V. K. Singh, Gold grating assisted SPR based D-shaped single mode fiber for detection of liquid refractive index, Opt. Quant. Electron. 51 (2019) 296.

[7] P. T. Arasu, A. S. M. Noor, A.A. Shabaneh, M. H. Yaacob, H. N. Lim, M. A. Mahdi, Fiber Bragg grating assisted surface plasmon resonance sensor with graphene oxide sensing layer, Opt. Commun. 380 (2016) 260-266.

[8] Q. H. Liu, B. Yan, J. J. Liu, U-shaped photonic quasi-crystal fiber sensor with high sensitivity based on surface plasmon resonance, Appl Phys Express. 12 (5) (2019) 052014.

[9] M. R. Hasan, S. Akter, A. A. Rifat, S. Rana, S. A. Ali, Highly Sensitive Gold-Coated Photonic Crystal Fiber Biosensor Based on Surface Plasmon Resonance. Photonics 4 (2017), 18.

[10] J. L. Han, E. X. Liu, J. J. Liu, Circular gradient-diameter photonic crystal fiber with large mode area and low bending loss, J. Opt. Soc. Am. A 36 (4) (2019) 533-539.

[11] Z. Huo, E. X. Liu, J. J. Liu, Hollow-core photonic quasicrystal fiber with high birefringence and ultra-low nonlinearity, Chin. Opt. Lett. 18 (3) (2020) 030603.

[12] E. X. Liu, S. W. Liang, J. J. Liu, Double-cladding structure dependence of guiding characteristics in six-fold symmetric photonic quasi-crystal fiber, Superlattice Microst. 130 (2019) 61-67.

[13] X. X. Wang, J. K. Zhu, X. L. Wen, X. X. Wu, Y. Wu, Y. W. Su, H. Tong, Y.P. Qi, H. Yang, Wide range refractive index sensor based on a coupled structure of Au nanocubes and Au film, Opt. Mater. Express 9 (7) (2019) 3079-3088. 
[14] M. Piliarik, H. Vaisocheróa, J. Homola, A new surface plasmon resonance sensor for high-throughput screening applications, Biosens. Bioelectron. 20 (10) (2005) 2104-2110.

[15] G. W. An, S. G. Li, X. Yan, X. N. Zhang, Z. Y. Yuan, H. Y. Wang, Y. N. Zhang, X. P. Hao, Y. N. Shao, Z. C. Han, Extra-broad photonic crystal fiber refractive index sensor based on surface plasmon resonance, Plasmonics. 12 (2) (2017) 465-471.

[16] A. K. Paul, A. K. Sarkar, A. Khaleque, Dual-core photonic crystal fiber plasmonic refractive index sensor: a numerical analysis, Photonic Sens. 9 (2) (2019) 151-161.

[17] K. Tong, F. C. Wang, M.T. Wang, P. Dang, Y.X. Wang, J.R. Sun, D-Shaped photonic crystal fiber biosensor based on silver-graphene, Optik. 168 (2018) 467-474.

[18] J. N. Dash, R. Jha, Highly sensitive D shaped PCF sensor based on SPR for near IR, Opt Quant. Electron. 48 (2) (2016) 137.

[19] M. Hautakorpi, M. Mattinen, H. Ludvigsen, Surface plasmon resonance sensor based on three hole microstructured optical fiber, Opt. Express 16 (12) (2008) 8427-8432.

[20] F. N. Zha, J. S. Li, P. J. Sun, H. J. Ma, Highly sensitive selectively coated D-shape photonic crystal fibers for surface plasmon resonance sensing, Phys. Lett. A 383 (15) (2019) 1825-1830.

[21] E. X. Liu, W. Tan, B. Yan, J. L. Xie, R. Ge, J. J. Liu, Robust transmission of orbital angular momentum mode based on a dual-cladding photonic quasi-crystal fiber, J. Phys. D Appl. Phys. 52 (2019) 325110.

[22] M. A. Mahfuz, M. R. Hasan, M. R. Momato, A. Masud., S. Akter, Asymmetrical photonic crystal fiber based plasmonic sensor using the lower birefringence peak method. OSA Continuum 2 (2019) 1713-1725.

[23] N. Luan, R. Wang, W. Lv; J.Yao, Surface plasmon resonance sensor based on D-shaped microstructured optical fiber with hollow core. Opt. Express. 23.(2015) 8576-8582.

[24] E. Haque, M. A. Hossain, Y. Namihira, F. Ahmed, Microchannel based plasmonic refractive index sensor for low refractive index detection, Appl. Opt. 58 (6) (2019) 1547-1554.

[25] L. Chao, W. Jianwei, J. Xin, W. Famei, Y. Lin, L. Jingwei, F. Guanglai, L. Xianli, L. Qiang, S. Tao, Ch. Paul $\mathrm{K}$, Near-infrared surface plasmon resonance sensor based on photonic crystal fiber with big open rings. Optik. 207. (2020) 164466.

[26] M. H. Kh. Anik, S.M. R. Islam, H. Talukder, S. Mahmud, M.I. A. Isti, A. Sadeghi-niaraki, K. Kwak, Sh. K. Biswas, A highly sensitive quadruple D-shaped open channel photonic crystal fiber plasmonic sensor: A comparative study on materials effect. Results in Physics. 23. (2021) 104050.

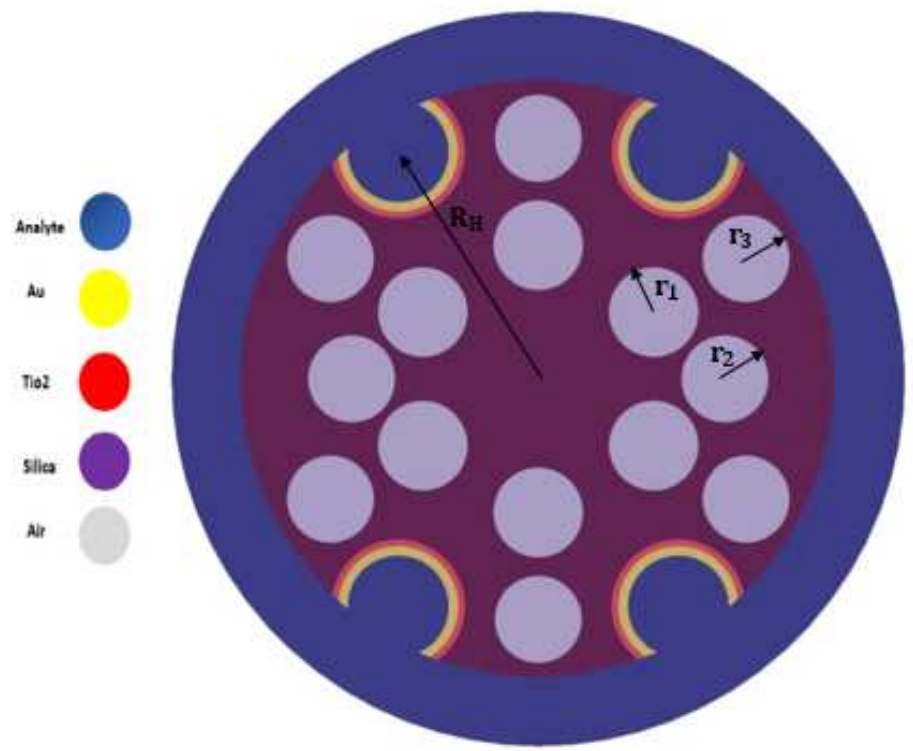


Fig. 1. Two-dimensional cross-section view of the proposed photonic crystal fiber (PCF) sensor with $\mathrm{R}_{\mathrm{H}}=4 \mu \mathrm{m}, \mathrm{r}_{1}=$ $0.67 \mu \mathrm{m}$.

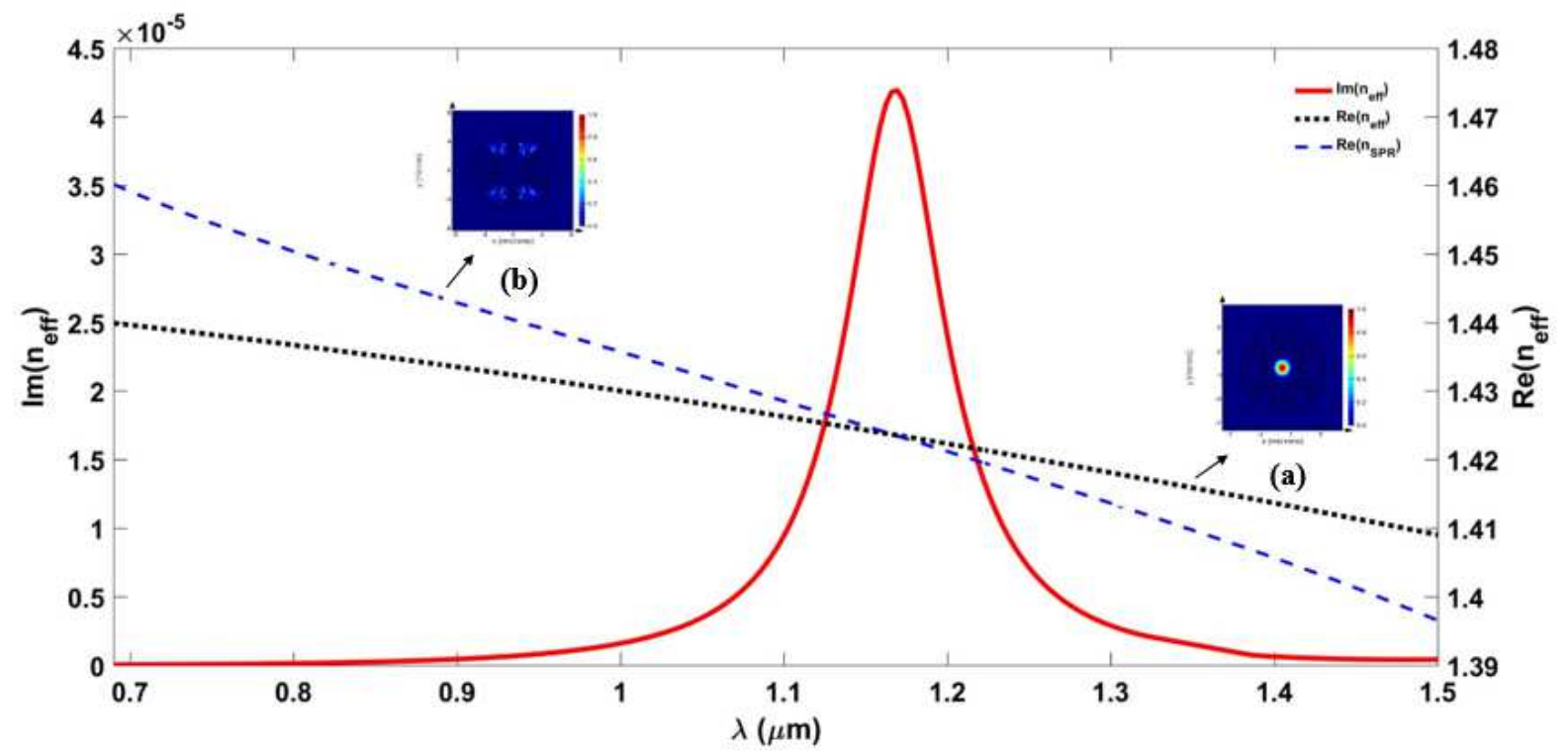

Fig. 2. Dispersion of the fundamental and SPR guided modes. Dotted and solid curves show the dispersion of real and imaginary part of fundamental mode, respectively and dashed curve shows the dispersion SPR mode. Inset a (b) displays the profile of electric fields for fundamental core (SPR) mode denoted by arrow for analyte RI of $1.43, t_{\mathrm{Au}}$ $=40 \mathrm{~nm}$ and $\mathrm{r}_{1}=0.67 \mu \mathrm{m}$.

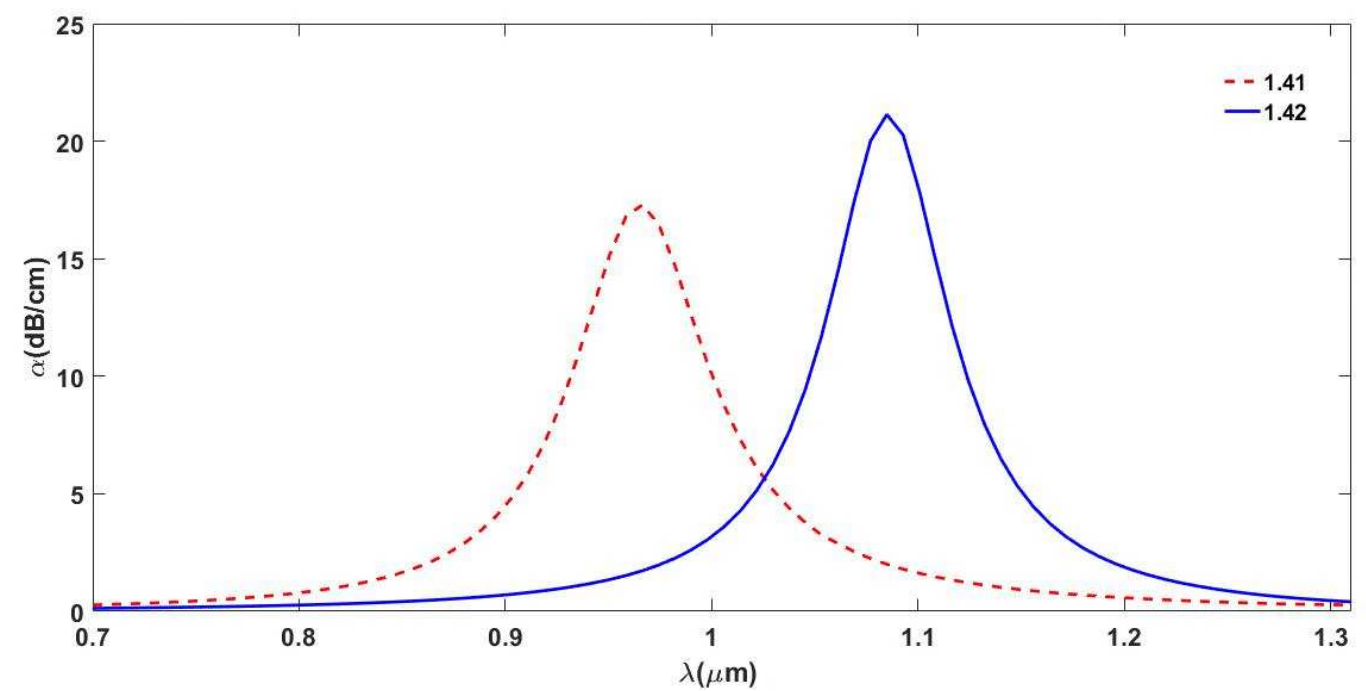

Fig. 3. Loss spectrum of proposed sensor for two analytes, where $t_{\mathrm{TiO}_{2}}=12 \mathrm{~nm}, \mathrm{t}_{\mathrm{Au}}=40 \mathrm{~nm}$ and $\mathrm{r}_{1}=0.65 \mu \mathrm{m}$ 


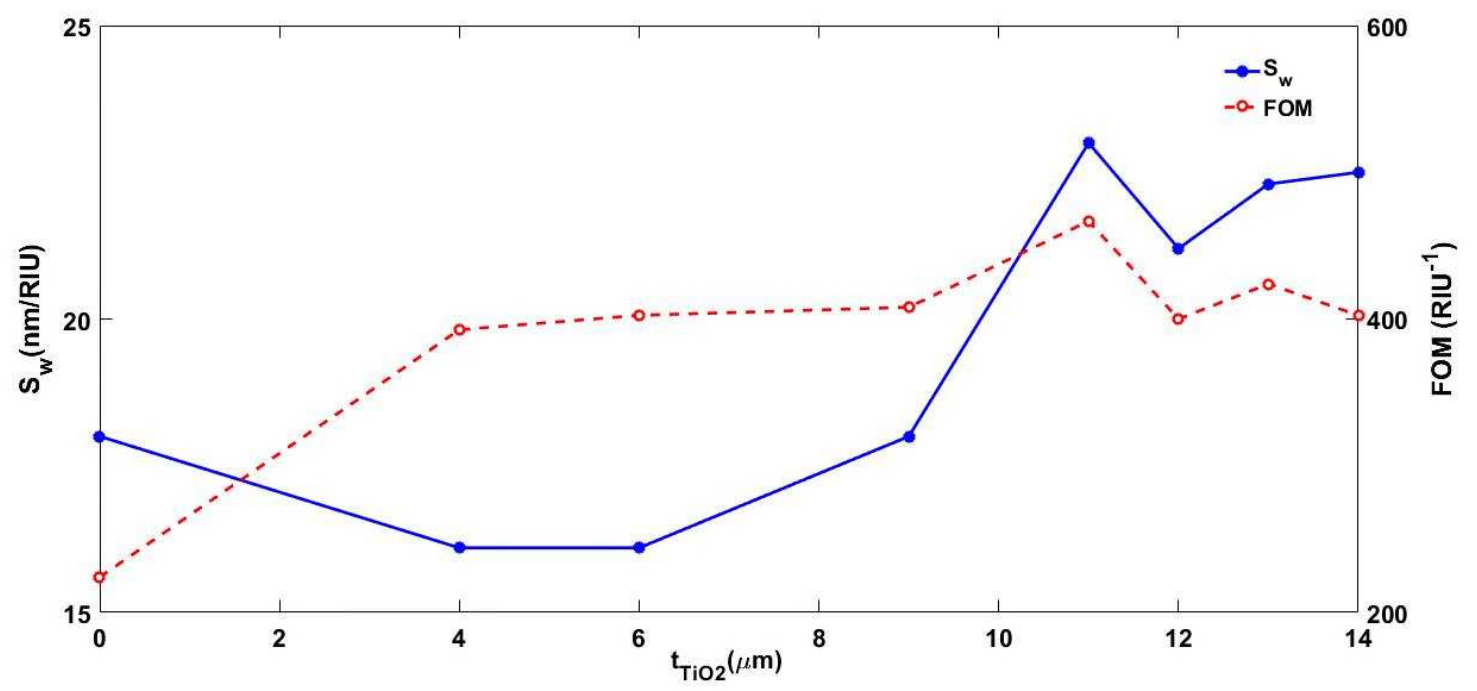

Fig. 4. The wavelength sensitivity and $\mathrm{FOM}$ of proposed sensor for different $\mathrm{TiO}_{2}$ thicknesses.

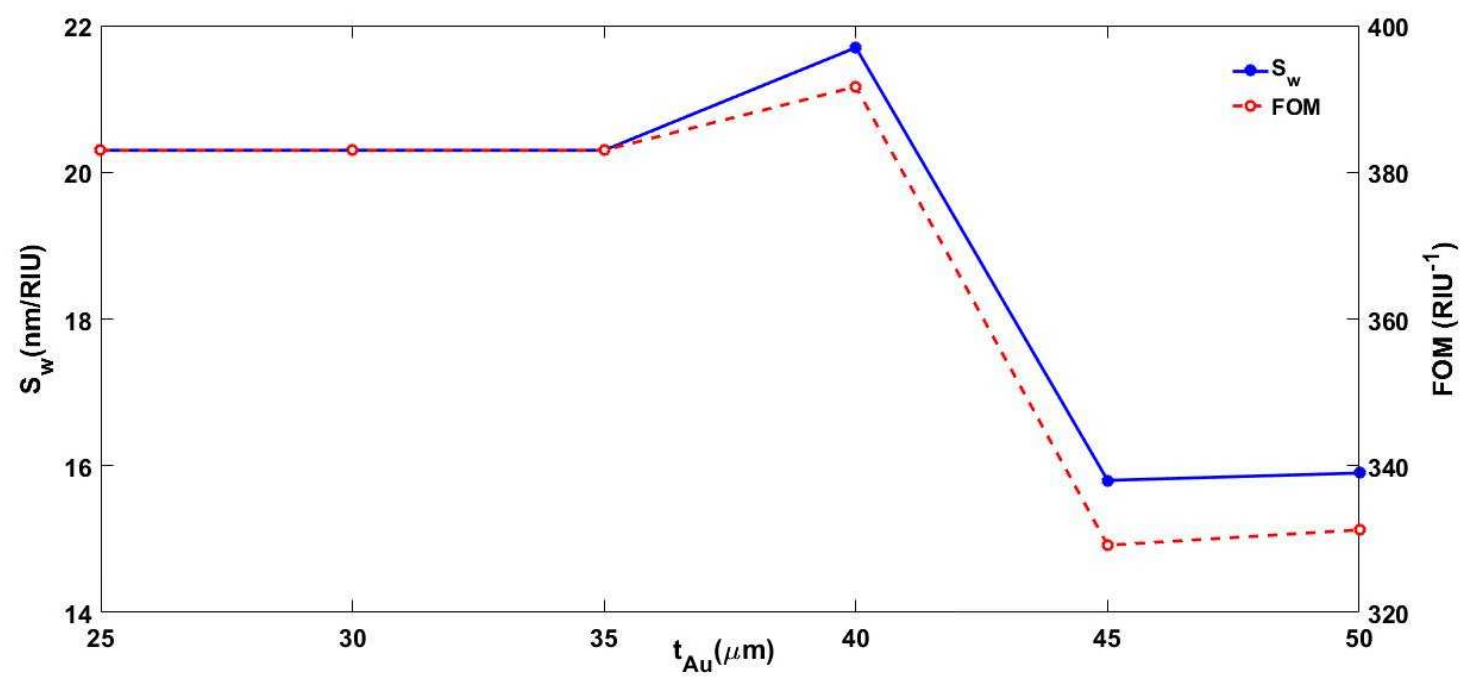

Fig. 5. The wavelength sensitivity and FOM for different Au layer thicknesses. 

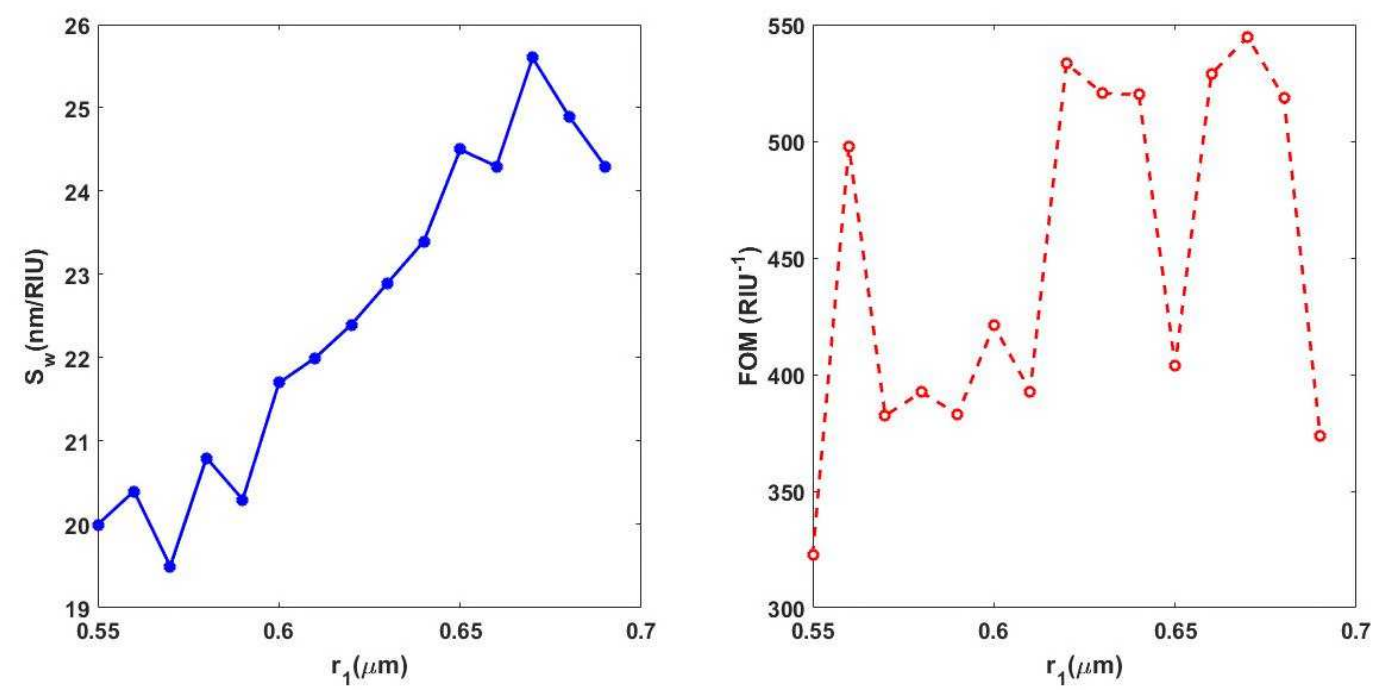

Fig.6. (a). Calculated wavelength sensitivity (left) and FOM (right) for different first air hole radii.

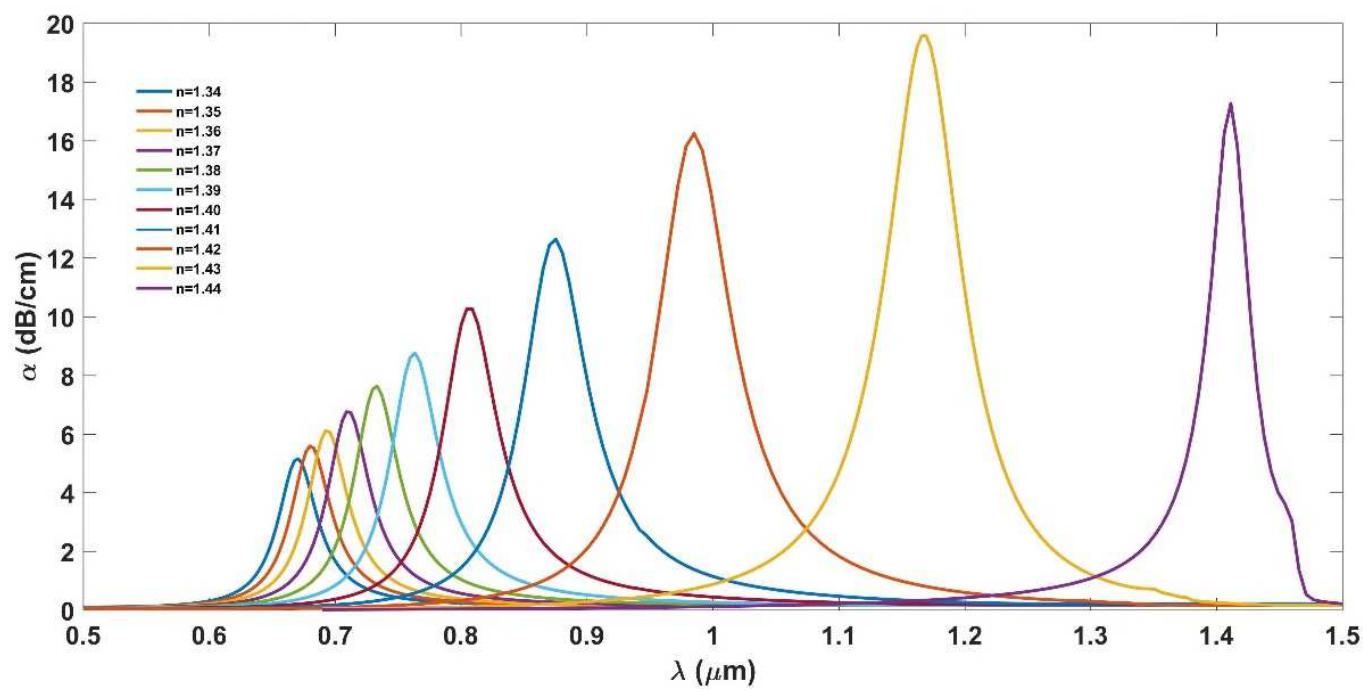

Fig. 7. Confinement loss spectra for analyte RIs variation from 1.34 to 1.44 . 

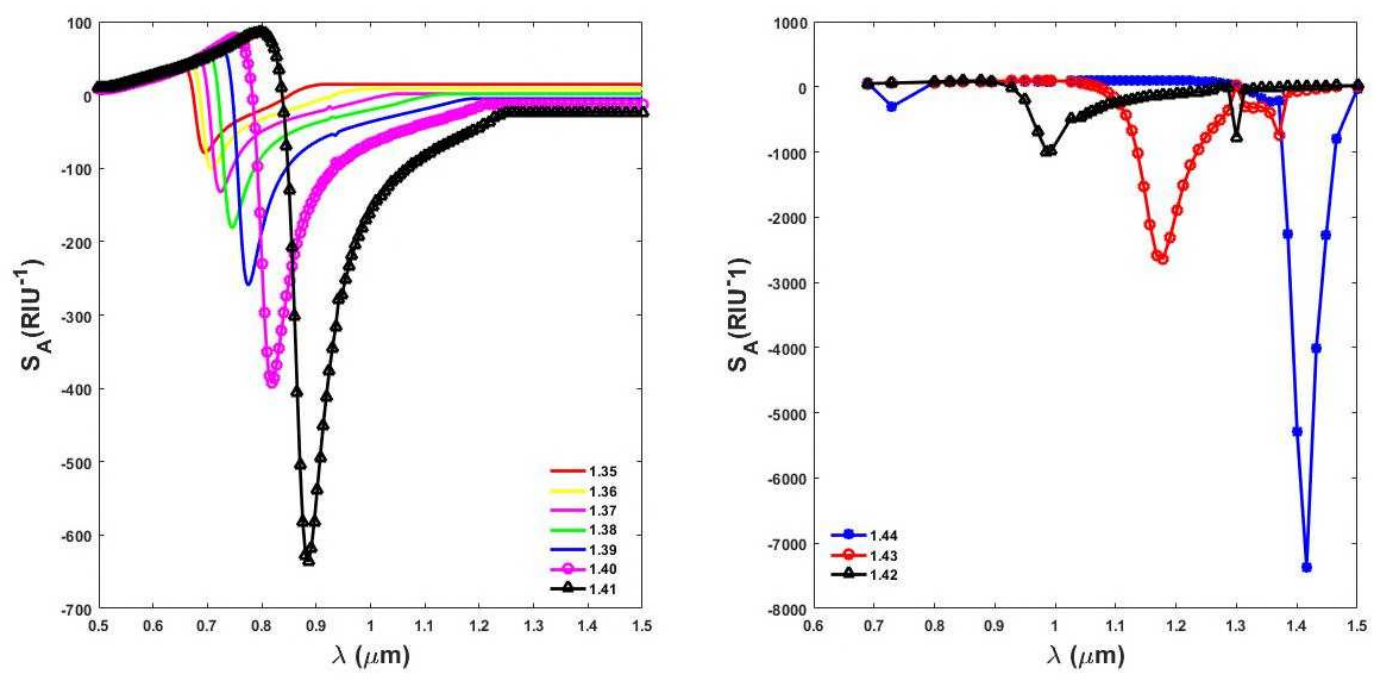

Fig. 8. Spectrum of the amplitude sensitivity of the proposed sensor with optimized parameters (left) RI=1.35-1.41, (right) RI=1.42-1.44.

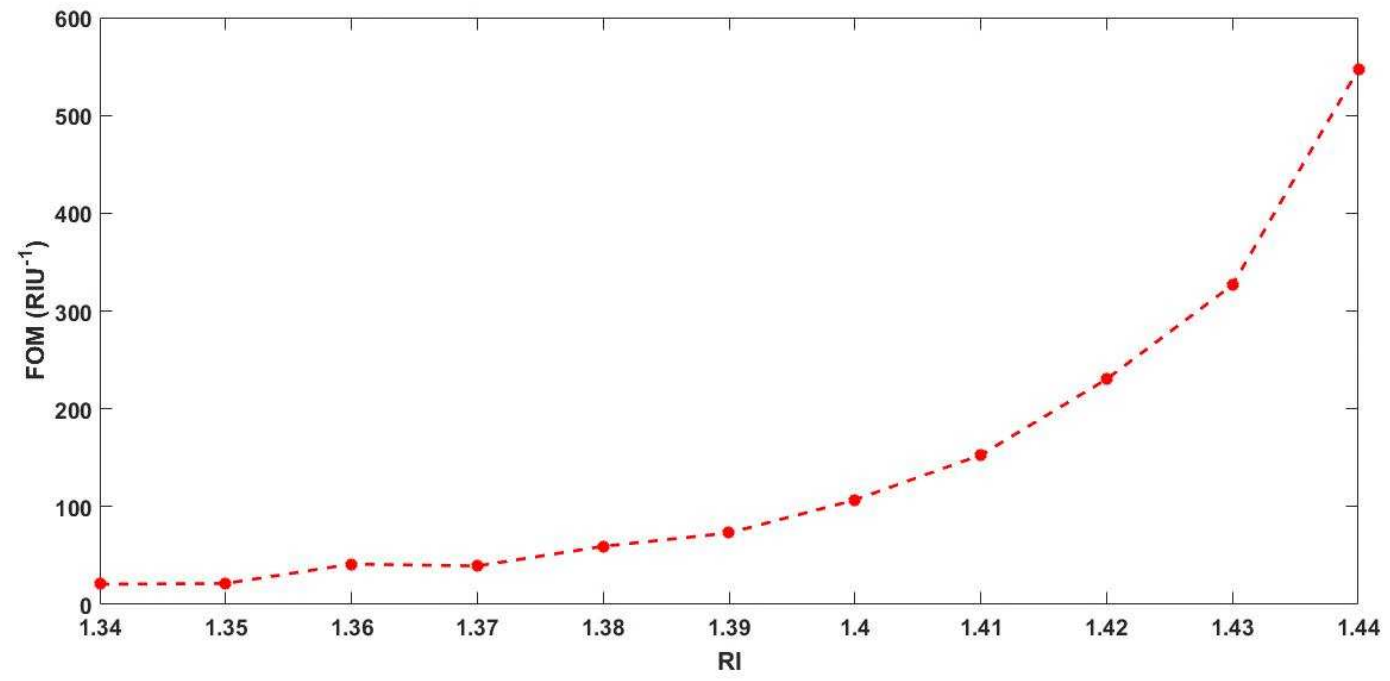

Fig. 9. FOM values of the proposed sensor for different analyte refractive indices. 


\section{Declaration of interests}

$\bigotimes$ The authors declare that they have no known competing financial interests or personal relationships that could have appeared to influence the work reported in this paper. 